\title{
Surface Plasmon resonance sensors for disease diagnosis
}

\begin{abstract}
This review paper presents the recent process in optical biosensors based on surface plasmon resonance (SPR). In particular, it will be focused on the optical sensors that employ the change of refractive index as the sensing transduction signal. The performance advantageous and disadvantageous will be given to the description of optical sensors structure and their respective experimental configurations. The application disease diagnosis will be presented. And future expectations of SPR biosensor are also discussed.
\end{abstract}

Keywords: detection limit, optical fiber, prism, surface plasmon resonance, sensitivity
Volume 3 Issue 5 - 2017

Tan Tai Nguyen

Department of Materials Science, Tra Vinh University,Vietnam

Correspondence: Tan Tai Nguyen, Department of Materials Science, School of Applied Chemistry, Tra Vinh University, Vietnam, Tel +84 2943855246 (Ext: 393),

Email nttai60@tvu.edu.vn

Received: December 14, 2017| Published: December 20, 2017

\section{Introduction}

Most of the optical sensors based on surface plasmon resonance (SPR) are imitated from two main configurations. Firstly, in the Kretschmann- Raether configuration, a thin metal layer is sandwiched between the prism and the air. Otherwise, the Otto configuration, there is a gap between the metal and the prism. However, the KretschmannRaether geometry is more conventional scheme to generate the surface plasmon wave in sensing technique. ${ }^{1}$ The incident light wave is also transverse magnetic wave (TM wave) that can be existed on the metal-air interface and used to excite the SPR under the boundary condition. ${ }^{2}$ Most of optical excitation of surface plasmon is based on prism, ${ }^{3}$ waveguide, ${ }^{4}$ optical fiber ${ }^{5}$ and grating. ${ }^{6}$ The SPR wave is described as an electromagnetic wave propagating along the interface between metal and dielectric medium. For three layers system, glass/ metallic/analyzed solution, the propagation constant $(\beta)$ of the surface plasmon wave propagated along the metal-analyze solution interface and the angular frequency, which can be described in the following

$$
\beta=\frac{\omega}{c}\left(\frac{\varepsilon_{b m} \varepsilon_{S}}{\varepsilon_{b m}+\varepsilon_{S}}\right)^{1 / 2}
$$

Where $\varepsilon_{b m}$ and $\varepsilon_{s}$ are the dielectric constants of the metal and the analyze solution, respectively. $\omega$ is the angular frequency of the incident light and $c$ is the speed of laser light. In the prism coupling configuration as depicted in Figure 1, an incident light wave passes through a high refractive index prism and then some portion of the light is reflected at the prism - metal interface. The evanescent wave will be generated by the coming laser light and penetrates into the thin metal film. The propagation constant of the evanescent wave shows below

$$
k_{x}=\frac{\omega}{c} n_{p} \sin \theta
$$

Where $\theta$ is the incident angle of the light, $n_{p}$ is the refractive index of the prism. The SPR will be generated by given the proper thickness of metal film and the resonance angle of light. Thus, the propagation constant of the evanescent wave will match that of the surface plasmon wave as shown below

$$
k_{x}=\frac{\omega}{c} n_{p} \sin \theta_{\text {res }}=\frac{\omega}{c}\left(\frac{\varepsilon_{b m} \varepsilon_{S}}{\varepsilon_{b m}+\varepsilon_{S}}\right)^{1 / 2}
$$

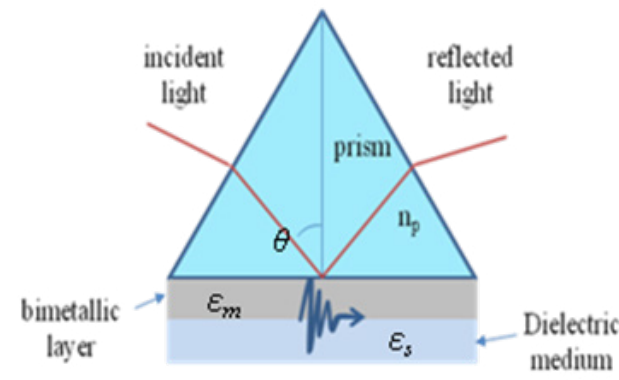

Figure I The schematic of the SPR sensor based on prism.

\section{Disease diagnosis based on SPR biosensor}

The SPR sensors have been developed for disease diagnosis applications by detection of some proteins, enzyme or antigen. This work has been focused on the detection and classification of the bioelements mentioned. An operation of the SPR sensors for examination the measurement of the target biomolecules can be classified in four steps including (1) incubate or capture the first targets, (2) inject the different concentration of the second binding targets, (3) perform the data, (4) regenerate the sensor. There are numerous scopes in SPR biosensors such as immunoassays including competitive immunoassay, noncompetitive immunoassay, heterogeneous immunoassay, homogeneous immunoassay via the antigensantibody interaction, chaperones in study interaction between protein and protein, screening for new target to understand the gene expression's mechanisms and signal transduction for different binding interactions. ${ }^{7}$ The detection limit of SPR biosensor in a particular measurements is strongly depended on the size, concentration and binding characteristic of targets. ${ }^{8}$ Recently, SPR biosensor has been used to investigate the absorption and desorption of IgG to the sensing surface with modification of protein $\mathrm{A},{ }^{9}$ monitoring the effect of glucose, lactate and human $\operatorname{IgG},{ }^{10}$ self - assembled using anti-human ferritin monoclonal antibodies for specific binding of antigens, ${ }^{11}$ measuring the immobilization of PSA-ACT mAb and PSA-ATC complex on the PSA sensor surface, ${ }^{12}$ display detection of different concentration of fibrinogen..$^{13}$ Addition to enhance the absorption of biomelecule or enzyme, the SPR sensing surface will be modified by thin polymer layer. ${ }^{14,15}$ Moreover, the covalent binding between antigen and antibody has also a powerful method for biosensors such as detection of metamphetamine, detection of low molecular weight 
domoic acid, medical diagnostics of human hepatitis B virus (hHBV), assay of the different poliovirus types. ${ }^{16-20}$ Another important field is study an immobilization of tTGas relating to the $\mathrm{Ca}^{2+}$ concentration, which is so important in human health. ${ }^{21}$ The SPR biosensors offers so sensitive technique, high selectivity and good regeneration in application.

\section{Future expectations of SPR biosensor}

Most of SPR biosensor based on prism with $\mathrm{Au}$ deposition on the sensor surface have been widely used with high resoution, multifunctionalization groups on the sensing surface, reusability and high stability. However, it is too expensive and abundant, leading difficulty in movement and opperation. Thus, focusing on finding and combination of different materials to generate new structure of sensor with high resolution and cost-effective is too specific important for future researches. In paricular, it will be focused on design and simulation the sensor's properties by using the multilayer of different materials such as $\mathrm{ZnO}, \mathrm{TiO}_{2}$, graphene to enhance the multilayer's properties based on specific properties of each material for pratical fabrication. Moreover, the regeneration of the sensing surface is also new trend for reseasrch.

\section{Conclusion}

The applications of SPR biosensor used are never be limited due to rapid development of biosensor. Most of researches are now focused on an improvement of their sensitivity, detection limit and operating refractive index range. We have seen that the SPR biosensor is used for disease diagnosis and extending their application for heavy metal detection, gas detection in future. In addition, there are many challenges to develop the new structure of biosensors. However, SPR biosensor is still the most convenient device providing easy operation, fast and specific detection in real-time manner.

\section{Acknowledgements}

None.

\section{Conflict of interest}

The author declares no conflict of interest.

\section{References}

1. Otto A. Excitation of nonradiative surface plasmon waves in silver by the method frustrated total reflection. Zeitschrift fur Physik. 1968;216(4):398-410.

2. Homola J, Yee SS. Surface plasmon resonance sensor based on planar light pipe: theoretical optimization analysis. Sensors and Actuators B. 1996;37(3):145-150.

3. Patskovsky S, Kabashin AV, Meunier M, et al. Near-infrared surface plasmon resonance sensing on a silicon platform. Sensors and Actuators B. 2004;97(2-3):409-414.

4. Dostalek J, Ctyroky J, Homola J, et al. Surface plasmon resonance biosensor based on integrated optical waveguide. Sensors and Actuators B. 2001;76(1-3):8-12.
5. Xu Y, Cottenden A, Jones NB. An approximate theoretical model of surface plasmon resonance optical waveguide and fibre-optic sensor. Optical and Quantum Electronics. 2005;37(12):1129-1140.

6. Hu C. Surface plasmon resonance sensor based on diffraction grating with high sensitivity and high resolution. Optik. 2011;122(21):18811884.

7. Szabo A, Stolz L, Granzow R. Surface plasmon resonance and its use in biomolecular interaction analysis (BIA). Current opinion in Structural biology. 1995;5(5):699-705.

8. Homola J. Present and future of surface plasmon resonance biosensors. Anal Bioanal Chem. 2003;377(3):528-539.

9. Tubb AJC, Payne FP, Millington RB, et al. Single mode optical fiber surface wave chemical sensor. Sensors and Actuators B. 1997;41(13):71-79.

10. Suzuki M, Nakashima Y, Mori Y. SPR immunosensor intergrated two miniature enzyme sensors. Sensors and Actuators B. 1999;54(1-2):176181.

11. Chou SF, Hsu WL, Hwang JM, et al. Development of an immunosensor for human ferritin, a nospecific tumor marker, based on surface plasmon resonance. Biosens Bioelectron. 2004;19(9):999-1005.

12. Hyun Soo Jang, Kwang No Park, Chang Duk Kang, et al. Optical fiber SPR biosensor with sandwich assy for the detection of prostate specific antigen. Optics communication. 2009;282(14):2827-2830.

13. Wang R, Lajevardi-Khosh A, Choi S, et al. Regenerative surface plasmon resonance (SPR) biosensor: Real-time measurement of fibrinogen in undiluted human serum using the competitive adsorption of proteins. Biosens Bioelectron. 2011;28(1):304-307.

14. Green RJ, Davies J, Davies MC, et al. Surface plasmon resonance for real time in situ analysis of protein adsorption to polymer surfaces. Biomaterials. 1997;18(5):405-413.

15. Wangkam T, Srikhirin T, Wanachantararak P, et al. Investigation of enzyme reaction by surface plasmon resonance (SPR) technique. Sensors and Actuators B. 2009;139(2):274-279.

16. Sakai G, Nakata $S$, Uda T, et al. Hightly selective and sensitive SPR immunosensor for detection of metamphetamine. Electrochimica Acta. 1999;44(21-22):3849-3854.

17. Yu Q, Chen S, Taylor AD, et al. Detection of low molecular weight domoic acid using surface plasmon resonance sensor. Sensors and Actuators B. 2005;107(1):193-201.

18. Chung JW, Kim SD, Bernhardt R, et al. Application of SPR biosensor for medical diagnostics of human hepatitis B virus (hHBV). Sensors and Actuators B. 2005;111-112:416-422.

19. Noah NM, Alam S, Sadik OA. Detection of inducible nitric oxide synthase using a suit of electrochemical fluorescence, and surface plasmon resonance biosensors. Analytical Biochemistry. 2011;413(2):157-163.

20. Manin C, Naville S, Gueugnon M, et al. Method for the simultaneous assay of the different poliovirus types using surface plasmon resonance technology. Vaccine. 2013;31(7):1034-1039.

21. Nikitin PI, Beloglazov AA, Kochergin VE, et al. Surface plasmon resonance interferometry for biological and chemical sensing. Sensors and Actuators B. 1999;54(1-2):43-50. 